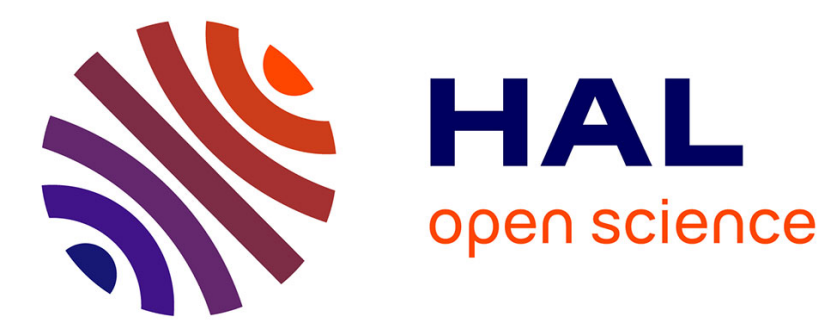

\title{
Modulated Metasurface Antennas with Enhanced Broadband Response
}

\author{
M. Faenzi, D. Gonzalez-Ovejero, S. Maci
}

\section{To cite this version:}

M. Faenzi, D. Gonzalez-Ovejero, S. Maci. Modulated Metasurface Antennas with Enhanced Broadband Response. 15th European Conference on Antennas and Propagation, EuCAP 2021, Mar 2021, Dusseldorf (virtual conference), Germany. pp.9411031, 10.23919/EuCAP51087.2021.9411031 . hal03249250

\section{HAL Id: hal-03249250 \\ https://hal.science/hal-03249250}

Submitted on 29 Nov 2021

HAL is a multi-disciplinary open access archive for the deposit and dissemination of scientific research documents, whether they are published or not. The documents may come from teaching and research institutions in France or abroad, or from public or private research centers.
L'archive ouverte pluridisciplinaire HAL, est destinée au dépôt et à la diffusion de documents scientifiques de niveau recherche, publiés ou non, émanant des établissements d'enseignement et de recherche français ou étrangers, des laboratoires publics ou privés. 


\title{
Modulated Metasurface Antennas with Enhanced Broadband Response
}

\author{
Marco Faenzi ${ }^{1}$, David González-Ovejero ${ }^{2}$, and Stefano Maci ${ }^{1}$ \\ ${ }^{1}$ Department of Information Engineering and Mathematics, University of Siena, Siena, Italy, \\ e-mail: \{faenzi, macis\}@diism.unisi.it \\ ${ }^{2}$ Univ Rennes, CNRS, IETR (Institut d'Électronique et de Télécommunications de Rennes), UMR 6164, 35000 Rennes, France \\ e-mail: david-gonzalez-ovejero@univ-rennes1.fr
}

\begin{abstract}
This paper deals with the crucial need of enhancing the gain-bandwidth of typically narrowband modulated metasurface (MTS) antennas. Despite their lowprofile and low-mass, modulated MTSs have typically been limited in terms of bandwidth. In broadside MTS antennas, this shortcoming stems from the progressive mismatch between the periodicity of the modulation (usually constant) and the wavenumber of the dispersive surface wave (SW). Here, an optimization scheme is introduced for the periodicity function, which is described as a piecewise monotonically increasing function along the antenna radius. The variable period leads to the generation of annular active regions, where the SW wavenumber matches the local periodicity, and a strong radiation occurs at the prescribed frequency. On the other hand, outside the annular region the SW weakly interacts with the MTS modulation. This works shows that the proposed method can considerably extend the bandwidth of MTS antennas.
\end{abstract}

Index Terms-metasurface antennas, broadband, leaky waves, surface waves.

\section{INTRODUCTION}

Aperture antennas based on modulated metasurfaces (MTSs) [1]-[10] have recently received considerable attention from the antenna community. In this class of antennas, radiation arises from the beating between a TM surface wave (SW) and a periodically modulated impedance boundary condition (IBC). This way, the -1 Floquet mode can enter the visible region, becoming radiative. The main advantages of modulated MTS antennas are their lowprofile and low mass, a simple fabrication by PCB technology [1]-[3] or additive manufacturing [4] and the absence of protruding feeds, as opposed to reflect-arrays and transmit-arrays. The IBC modulation is typically achieved by a dense textured layer composed of many small metal patches printed on a grounded dielectric slab [1]-[3]. These patches are arranged on a periodic lattice, and they take different shapes and dimensions to provide the requested impedance modulation.

Modulated MTS antennas are especially appealing for space, where strict mass and volume constraints apply. Moreover, they have demonstrated the capability of coping with a variety of challenging requirements such as contoured beams [2]-[3], high gains [2]-[3],[5], multi frequency operation [6]-[7], dual polarization [8]-[9], and multiple beams [10]. It is important to note that such features do not alter the MTS low mass and low envelope; a careful design of the patch texture suffices to achieve an accurate control of the aperture field in terms of amplitude, phase and polarization.

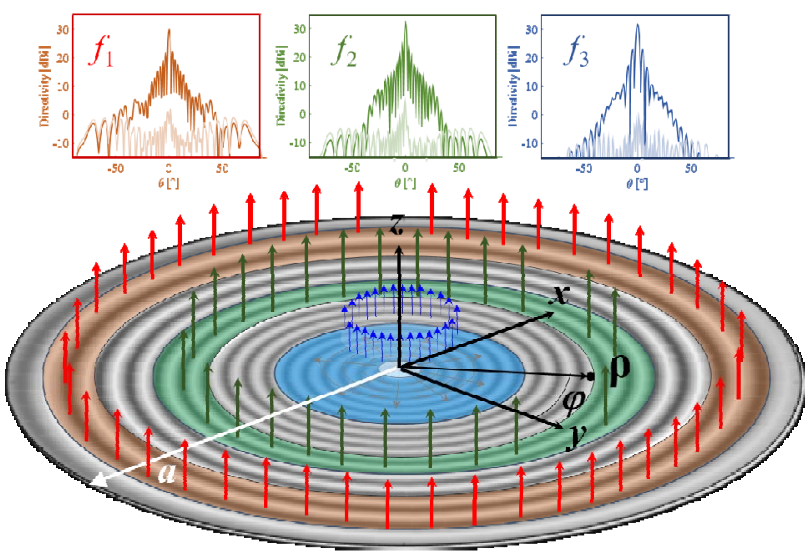

Fig. 1. Reference coordinate system and active regions (colored areas) at three different frequencies $\left(f_{1}<f_{2}<f_{3}\right)$, which radiation patterns are shown in the top insets.

Nonetheless, MTS apertures are typically constrained by a limited gain-bandwidth. Indeed, it was shown in [11] that broadside apertures provide relative bandwidths $\left(\Delta f / f_{0}\right)$ ranging from $3 \%$ to $9 \%$ for decreasing gains going from 40 to $28.5 \mathrm{dBi}$. Such detrimental effect originates from a progressive mismatch between the SW wavelength $\lambda_{s w}$ and the periodicity $d$ of the modulation as frequency shifts from the central frequency, $f_{0}$. The SW wavelength variation is mainly due to the dispersive behaviour of the dielectric substrate, which causes a progressive beam depointing and thus a rapid gain reduction.

A method was proposed in [12] for enhancing the gainbandwidth of circular apertures. It is based on adopting a radially-dependent periodicity function $d(\rho)$ that grows exponentially with the radius. Then, as frequency shifts from lower to higher frequencies, the matching between $\lambda_{\text {sw }}$ and $d(\rho)$ occurs in an active annular region that progressively shifts from the rim of the antenna to its center 
(see Fig. 1). However, the gain responses obtained in [12] still show a quite high fluctuation with respect to the average gain value. In this paper, we propose an optimization scheme to eliminate these gain fluctuations. To this end, a more general shape of $d(\rho)$ is considered in the optimization algorithm, which also manages the frequency $\omega_{p}$ at which the MTS texture is designed to obtain an efficient aperture illumination.

This paper is structured as follows. Section II presents the problem at hand and the initial algorithm setup. Section III details the algorithm scheme, and numerical results relevant to a specific broadband design are presented in section IV. Finally, conclusions are drawn in Section V.

\section{PROBLEM DESCRIPTION AND ALGORITHM SETUP}

The circular apertures dealt with are characterized by a radius $a$, while the texture lays at $z=0$ plane of a cylindrical reference system (see Fig. 1). This texture is modelled by a penetrable IBC as

$$
\mathbf{E}_{t}=\mathrm{j} \underline{\underline{\mathbf{X}}} \cdot \hat{\boldsymbol{z}} \times\left(\left.\mathbf{H}_{t}\right|_{z=0^{+}}-\left.\mathbf{H}_{t}\right|_{z=0^{-}}\right)
$$

where $\left.\boldsymbol{H}_{t}\right|_{z=0^{+}}$and $\left.\boldsymbol{H}_{t}\right|_{z=0^{-}}$are the tangential components of the magnetic field infinitesimaly close to the IBC for positive and negative $z$ values. $\mathbf{E}_{t}$ is the averaged tangential electric

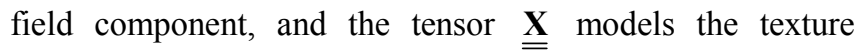
reactance. A generic point on the aperture is defined in cylindrical coordinates as $\rho=\rho \cos \varphi \hat{\mathbf{x}}+\rho \sin \varphi \hat{\mathbf{y}}$, and the modulation of $\underline{\underline{\mathbf{X}}}$ is written as

$$
\begin{array}{r}
\underline{\mathbf{X}}=\bar{X}_{\rho}\left\{\underline{\underline{\mathbf{I}}}+\left[\cos [\Phi(\rho)+\varphi]\left(m_{\rho}(\rho) \hat{\boldsymbol{\rho}} \hat{\boldsymbol{\rho}}-m_{\varphi}(\rho) \hat{\varphi} \hat{\varphi}\right)\right.\right. \\
\left.\left.-m_{\rho}(\rho) \sin [\Phi(\rho)+\varphi](\hat{\boldsymbol{\rho}} \hat{\boldsymbol{\varphi}}+\hat{\varphi} \hat{\boldsymbol{\rho}})\right]\right\}
\end{array}
$$

where $\bar{X}_{\rho}$ is the average reactance value, $m_{\rho}, m_{\varphi}$ are radius-dependent modulation indexes, and $\Phi(\rho)$

$$
\Phi(\rho)=\int_{0}^{\rho} \frac{2 \pi}{d\left(\rho^{\prime}\right)} d \rho^{\prime}+\Phi(0)
$$

is the phase of the modulation calculated from the periodicity function $d(\rho)$, where $\Phi(0)$ in (3) is a fixed initial phase. In this work, we adopt a function $d(\rho)$ that results from the optimization scheme in Fig. 2 to get rid of the gain fluctuations observed in [12].

The optimization process starts by selecting the desired bandwidth, delimited by the angular frequencies $\omega_{\min }$ and $\omega_{\max }$, and with $\omega_{0}$ center frequency. The grey box in Fig. 2 represents this step. The objective in-band average gain $G_{\text {ave }}$ is then selected and the aperture radius $a$ is found using the formula [13]

$$
G_{\text {ave }} \frac{\Delta f}{f_{0}} \approx 28.7 \frac{v_{g, a v e}}{c} \frac{a}{\lambda_{0}}<28.7 \frac{a}{\lambda_{0}}
$$

where $\lambda_{0}$ is the free space wavelength at $\omega_{0}$ and $v_{g \text {,ave }}$ is the integral average of the group velocity associated to $\bar{X}_{\rho}(\omega)$ within the objective frequency band. Of course, one may also determine $G_{\text {ave }}$ starting from a fixed radius.

The preliminary choice of the MTS substrate (in the following we use a Rogers RO3006 laminate with $\varepsilon_{\mathrm{r}}=6.15$ and thickness $h=0.635 \mathrm{~mm}$ ) affects the selection of the texture; we have adopted here elliptical elements. The definition of the texture allows one to fix the average reactance value $\bar{X}_{\rho}\left(\omega_{0}\right)$ at $f_{0}$, which is maintained constant at any optimizer iteration. Once $\bar{X}_{\rho}\left(\omega_{0}\right)$ has been defined, the dispersion of the wavenumber $\beta_{\text {sw }}$ of a SW propagating on the average texture reactance $\bar{X}_{\rho}(\omega)$ is characterized within the band of interest by using the full-wave solver in [15]. The obtained results are used to build the dispersion database (DB), shown as step A in Fig. 2.

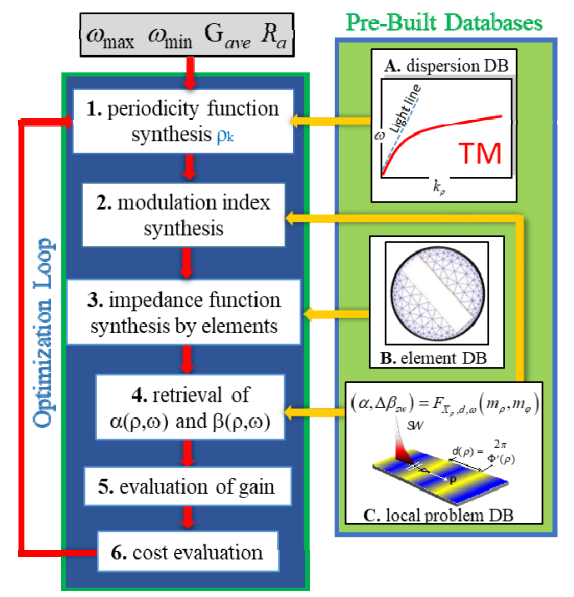

Fig. 2 Flow diagram for the optimization process.

A set of $N$ equispaced inband phase control frequencies $f_{n}$ are selected and the SW wavelengths $\lambda_{s w, n}=2 \pi / \beta_{s w, n}$ (obtained from dispersion curve) are associated to each frequency $f_{n}$. Each $\lambda_{s w, n}$ is then linked to a radial control point $\rho_{n}$ so to build the function $d(\rho)$. The $\rho_{n}$ ordering is done to preserve a monotonic growth of $d(\rho)$ for increasing $\rho$. Therefore, we assume that to every $f_{\mathrm{n}}$ corresponds the center of the $n$th active region located in $\rho_{\mathrm{n}}$. The operations above are carried out in step 1 in Fig. 2.

In order to get a flat gain response, it is also important to select an amplitude control frequency $\omega_{p}$ at which the amplitude of the -1 indexed Floquet is imposed as constant along $\rho$. In the optimization process, this frequency can vary within the design band. The coordinates $\rho_{\mathrm{n}}$ and the frequency $\omega_{\mathrm{p}}$ are iteratively managed by the algorithm using as control the value $C$ of a cost function calculated at the end of any iteration, and defined next. In the first iteration, radially equispaced control points $\rho_{\mathrm{n}}$ are assumed, and $\omega_{p}$ coincides with the middle frequency $\omega_{0}$. The cost function $C$ is defined as

$$
C=\sum_{i} \frac{\left|G\left(\omega_{i}\right)-G_{a v e}\right|}{G_{\text {ave }}} .
$$




\section{OPTIMIZATION ALGORITHM WORKFLOW}

Computing the cost function $C$ requires the evaluation of the inband gain $G(\omega)$ as [5]

$$
G(\omega)=\frac{8 \pi^{2}}{\lambda^{2}} \frac{\left|\int_{0}^{a} \sqrt{S(\rho, \omega)} e^{j \Psi(\rho, \omega)} \rho d \rho\right|^{2}}{\int_{0}^{a} S(\rho, \omega) \rho d \rho}
$$

$S(\rho, \omega)$ in (6) represents the surface power density distribution and $\Psi$ is the phase of the -1 mode in the adiabatic Floquet expansion [16] defined as

$$
\Psi(\rho, \omega)=\Phi(\rho)-\beta_{s w} \rho-\int_{0}^{\rho} \Delta \beta_{s w}\left(\rho^{\prime}\right) d \rho^{\prime}
$$

whre $\Delta \beta_{s w}$ represents a deviation from $\beta_{s w}$ due to the reactance modulation, and can be found by solving the local canonical problem [17], the phase $\Phi(\rho)$ is instead related to $d(\rho)$ through (3).

The gain $G$ depends on $\omega$ by means of $S(\rho, \omega)$ and $\Psi(\rho, \omega)$, which frequency dependence is due to the MTS dispersion. At lower frequencies (active rings are located in the vicinity of the rim), it is particularly important to obtain a uniform illumination of the aperture. To this end, the optimizer adaptively changes the frequency $\omega_{\mathrm{p}}$ at which a uniform profile of $S\left(\rho, \omega_{\mathrm{p}}\right)$ is imposed, with rapid zero dropping close to the source and the rim (see [12] for $S\left(\rho, \omega_{\mathrm{p}}\right)$ expression). Once $S\left(\rho, \omega_{\mathrm{p}}\right)$ has been defined, one can retrieve the local attenuation constant $\alpha\left(\rho, \omega_{\mathrm{p}}\right)$ for the -1 Floquet mode from

$$
\alpha\left(\rho, \omega_{p}\right)=\frac{\rho S\left(\rho, \omega_{p}\right) / 2}{\frac{1}{2 \pi} P_{s w}-\int_{0}^{\rho} S\left(\rho^{\prime}, \omega_{p}\right) \rho^{\prime} d \rho^{\prime}}
$$

where $P_{s w}$ is the power associated to the SW. Next, by solving the generalized local canonical problem that matches the local periodicity $d(\rho)$ and average reactance $\bar{X}_{\rho}$ [17] it is possible to find the modulation index functions (step 2 in Fig. 2) that locally match the profile of $\alpha$ in (8) (here we consider $m_{\rho}(\rho)$ equal to $m_{\varphi}(\rho)$, see Fig. 3). At this point, the reactance tensor $\underline{\underline{X}}\left(\rho, \omega_{p}\right)$ is completely characterized at frequency $\omega_{p}$.

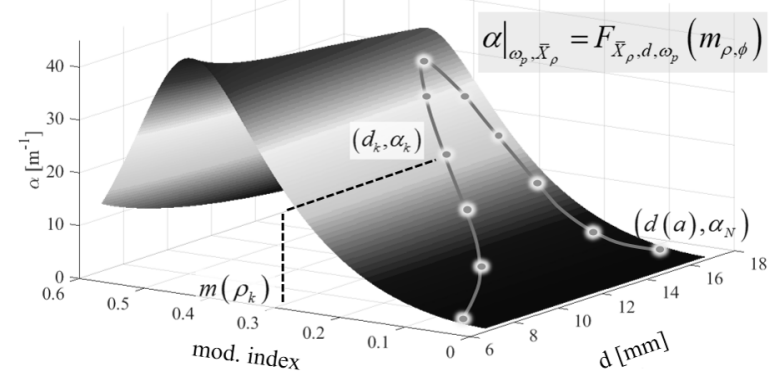

Fig. 3 The surface represents the solution of the canonical problem [17] for the attenuation constant $\alpha$ for a set of modulation indexes and periodicities at frequency $\omega_{\mathrm{p}}$. Local modulation index for any $\rho_{\mathrm{n}}\left(d_{\mathrm{n}}\right)$ is found by selecting on on the modulation index axis the value that provides the desired $\alpha\left(\rho_{\mathrm{n}}, \omega_{\mathrm{p}}\right)$.
The successive steps consist in determining $\Delta \beta_{s w}(\rho, \omega)$ and $\alpha(\rho, \omega)$ as functions of $\omega$. With $\Delta \beta_{s w}(\rho, \omega)$, one can obtain $\Psi(\rho, \omega)$, while $S(\rho, \omega)$ may be computed using $\alpha(\rho, \omega)$ in the inverse formula of $(8)$

$$
S(\rho, \omega)=2 \alpha(\rho, \omega) \frac{1}{\rho} \frac{P_{S W}}{2 \pi} \exp \left(-2 \int_{0}^{\rho} \alpha\left(\rho^{\prime}, \omega\right) d \rho^{\prime}\right) .
$$

Now, $G(\omega)$ is fully defined.

The calculation of $\Delta \beta_{s w}(\rho, \omega)$ and $\alpha(\rho, \omega)$ above, implies the characterization of the reactance tensor $\underline{\underline{X}}(\rho, \omega)$ for any frequency $\omega$. Knowing $\underline{\underline{X}}\left(\rho, \omega_{p}\right)$, we can synthesize the surface by metal elements (step 3 in Fig. 2). This operation is carried out using the periodic spectral MoM in [15], which uses Rao-Wilton-Glisson basis functions, to calculate a database that associates reactance values to the geometry of metallic patches. Such database (B in Fig. 2) is produced for any frequency $\omega$. Then, the surface synthesis is performed at $\omega_{p}$ by a least-square error (LSE) minimization for any unit cell, and the local reactance values are also stored for the other frequencies $\omega$. To speed-up the iteration, the surface synthesis is only performed along direction $\varphi=0$. Given the linear dependence of $\underline{=}$ on $\varphi$, the surface reactance can be easily reconstructed by algebraic relations for any $\varphi$ and $\omega$ pair.

At the end of this process we obtain a family of reactance curves which represents the radial variation of the reactance with respect to $\omega$, as represented in Fig. 4. By using these curves, it is easy to retrieve the actual frequency variation of the modulation indexes $m_{\rho}(\rho, \omega), m_{\varphi}(\rho, \omega)$ and the average reactance value $\bar{X}_{\rho}(\omega)$ of the synthesized reactance function.

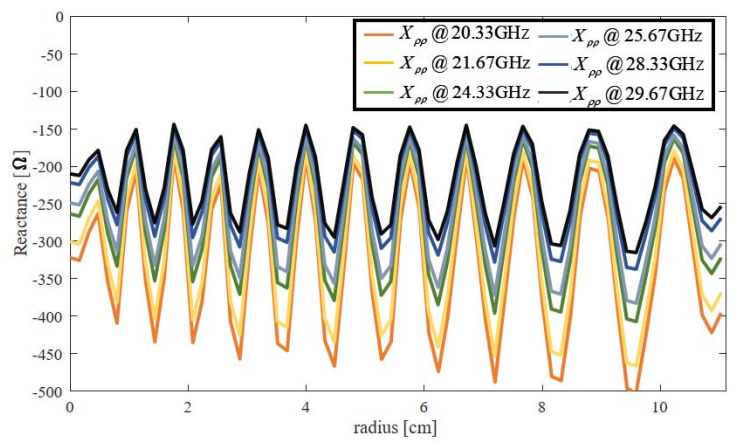

Fig. 4 Representation by colored curves of the $X_{\rho \rho}$ reactance profile variation at several different frequencies $\omega$ within the antenna bandwidth along $\varphi=0$.

The iteration is then carried on (step 4 in Fig. 2) by determining the relation between the sinthesyzed modulation indexes and average reactance and the leaky-wave parameters $\alpha(\rho, \omega)$ and $\Delta \beta_{s w}(\rho, \omega)$, which represent the perturbation on the SW wavenumber due to the modulation. This link is provided for a generic point $\left(\rho_{0}, \varphi_{0}\right)$ on the surface using the synthesized functions $m_{\rho}(\rho, \omega)$, $m_{\varphi}(\rho, \omega)$ and $\bar{X}_{\rho}(\omega)$ to set the generalized canonical problem [17] that locally matches the modulation features 
$m_{\rho}\left(\rho_{0}, \omega\right), \quad m_{\varphi}(\rho, \omega), d\left(\rho_{0}\right)$ for any $\omega$. Formally one can express this relation as

$$
\left(\alpha, \Delta \beta_{s w}\right)=F_{\bar{X}_{\rho}, d, \omega}\left(m_{\rho}, m_{\varphi}\right) .
$$

The information for $\alpha(\rho, \omega)$ and $\Delta \beta_{s w}(\rho, \omega)$ is stored in database $\mathrm{C}$, as shown in Fig. 2. At this point, one can proceed to the calculation of the gain versus frequency response (step 5 in Fig. 2) and of the cost function (step 6 in Fig. 2).

The proposed procedure is very fast, as the runtime for one iteration is around $1 \mathrm{~min}$ to evaluate the gain at 25 frequency points for a Ka-band antenna of radius $a=11.11 \mathrm{~cm}$ on an I7-Intel core machine.

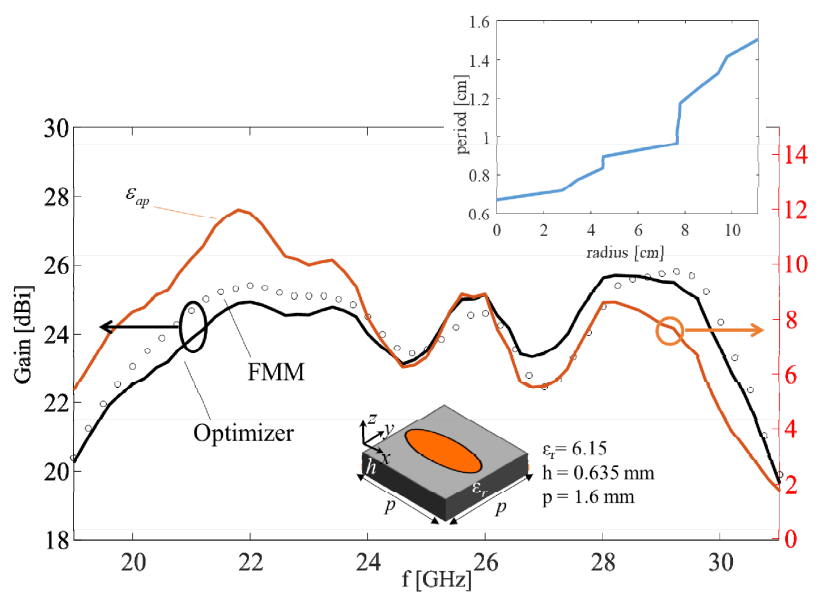

Fig. 5 Gain versus frequency responses for the $11.11 \mathrm{~cm}$ radius optimized antenna. The solid lines stand for full-wave analysis of the homogenized impedance with the MoM tool in [18], while the circular markers correspond to the FMM simulation [3] of the apertures synthesized by elliptical patches. The top inset shows the final periodicity as a function of the radial distance.

\section{NUMERICAL RESULTS}

The optimization scheme detailed in Sections II and III has been used to design a broadband aperture with radius $a=11.11 \mathrm{~cm}$. After selecting the upper and lower frequencies $f_{\max }=29.5 \mathrm{GHz}$ and $f_{\min }=20.5 \mathrm{GHz}$, we choose the average reactance value at center frequency $f_{0}=25 \mathrm{GHz}$ as $\bar{X}_{\rho}\left(\omega_{0}\right)=-260.7 \Omega$. Therefore, using (4) one can predict an in-band average gain $G_{a v e}=25.4 \mathrm{dBi}$. Fig. 5 presents the gain response of the aperture designed by the proposed optimization algorithm, which converged to the optimum solution in about one thousand iterations. The so-obtained layout has been tested by a full-wave MoM solver for the ideal MTS reactance [18] (solid lines in Fig. 5), and by a fast multipole method (FMM) [3] of the same aperture synthesized by elliptical patches elements arranged in a Cartesian lattice with unit cell side of $1.6 \mathrm{~mm}$ (circular markers in Fig. 5). The FMM exploits the analytical entire domain functions in [14]. The agreement between the two curves is excellent, and the calculated average gain value is close to the expected one. The oscillation of the gain response around $G_{\text {ave }}$ is also limited to around $\pm 1.5 \mathrm{~dB}$.
Besides the reduced gain oscillation, the bandwidth is considerably extended with respect to the prototype in [12], which is based on an exponential profile of the periodicity function $d(\rho)$. Finally, the inset in Fig. 5 represents the output optimal function for the periodicity function $d(\rho)$, whereas the optimized amplitude control frequency was $\omega_{p}=21.85 \mathrm{GHz}$.

\section{CONCLUSION}

A novel methodology has been introduced for the design of broadside modulated MTS antennas with a broadband response. The presented method relies on an optimization scheme, which outcome is a layout able to provide a flat gain response over broad bandwidths. An accurate formula for the gain-bandwidth product is also introduced to establish a bound for the performance of this kind of antennas. In the first instance, the gain bandwidth and the gain oscillations are controlled by adopting a general piecewise shape of the periodicity function $d(\rho)$. Each periodicity value is related to the surface wave (SW) wavelength that provides broadside pointing at a given frequency among a set of points in the band of interest. The radial position for each periodicity value is one of the optimization parameters. Second, the amplitude of the -1 mode is also controlled by imposing a quasi-constant profile at the frequency $\omega_{p}$, which is also adapted at each iteration of the optimization loop. This way, it is possible to design flat and low-mass apertures that are able to provide a very stable gain performance over considerably large bandwidths, overcoming a typical limitation for this class of antenna.

\section{REFERENCES}

[1] B. H. Fong, J. S. Colburn, J. J. Ottusch, J. L. Visher, and D. F. Sievenpiper, "Scalar and tensor holographic artificial impedance surfaces," IEEE Trans. Antennas Propag., vol. 58, no. 10, 435 pp. 3212-3221, Oct. 2010.

[2] G. Minatti, F. Caminita, E. Martini, M. Sabbadini, and S. Maci, "Synthesis of modulated-metasurface antennas with amplitude, phase, and polarization control," IEEE Trans. Antennas Propag., vol. 64, no. 9, pp. 3907-3919, Sep. 2016.

[3] M. Faenzi et al., "Metasurface Antennas: New models, applications and realizations," Sci. Rep., vol. 9, pp. 10178, 2019.

[4] D. González-Ovejero, N.Chahat, R. Sauleau, G. Chattopadhyay, S. Maci, and M. Ettorre, "Additive Manufactured Metal-Only Modulated Metasurface Antennas," IEEE Trans. Antennas Propag., vol. 66, no. 11, pp. 6106-6114, Nov. 2018.

[5] G. Minatti, E. Martini, and S. Maci, "Efficiency of metasurface antennas," IEEE Trans. Antennas Propag., vol. 65, no. 4, pp. 15321541, April 2017.

[6] M. Faenzi, D. González-Ovejero, F. Caminita, and S. Maci, "Dualband self-diplexed modulated metasurface antennas," in Proc. 12nd Eur. Conf. Antennas Propag., London, 2018.

[7] Y. Li, A. Li, T. Cui, and D. F. Sievenpiper, "Multiwavelength multiplexing hologram designed using impedance metasurfaces," IEEE Trans. Antennas Propag., vol. 66, no. 11, pp. 6408-6413, Nov. 2018.

[8] A. T. Pereda et al., "Dual circularly polarized broadside beam metasurface antenna," IEEE Trans. Antennas Propag., vol. 64, no. 7, pp. 2944-2953, Jul. 2016. 
[9] M. Li, S. Xiao, and D. F. Sievenpiper, "Polarization-insensitive holographic surfaces with broadside radiation," IEEE Trans. Antennas Propag., vol. 64, no. 12, pp. 5272-5280, Dec. 2016.

[10] D. González-Ovejero, G. Minatti, G. Chattopadhyay, and S. Maci, "Multibeam by metasurface antennas," IEEE Trans. Antennas Propag., vol. 65, no. 6, pp. 2923-2930, Jun. 2017.

[11] G. Minatti, M. Faenzi, M. Sabbadini, and S. Maci, "Bandwidth of gain in metasurface antennas," IEEE Trans. Antennas Propag., vol. 65, no. 6, pp. 2836-2842, Jun. 2017.

[12] M. Faenzi, D. González-Ovejero, and S. Maci, "Wideband active region metasurface antennas," IEEE Trans. Antennas Propag., vol. 68, no. 3, pp. 1261-1272, Mar. 2020.

[13] M. Faenzi, D. González-Ovejero and S. Maci, "Flat gain broad-band metasurface antennas," IEEE Trans. Antennas Propag., in press.

[14] M. Mencagli, E. Martini, and S. Maci, "Surface wave dispersion for anisotropic metasurfaces constituted by elliptical patches," IEEE Trans. Antennas Propag., vol. 63, no. 7, pp. 2992-3003, Jul. 2015.

[15] S. Maci and A. Cucini, "FSS-based EBG surface" in Electromagnetic Metamaterials: Physics and Engineering Aspects, N. Engheta, R. Ziolkowski Eds., Hoboken, NJ:Wiley, 2006.

[16] G. Minatti, F. Caminita, E. Martini, and S. Maci, "Flat optics for leaky-waves on modulated metasurfaces: Adiabatic Floquet-wave analysis," IEEE Trans. Antennas Propag., vol. 6, no. 9, pp. 38963906, Sep. 2016.

[17] F. Caminita and S. Maci, "New wine in old barrels: The use of the Oliner's method in metasurface antenna design," 44th Eur. Microw. Conf., Rome, 2014.

[18] D. González-Ovejero and S. Maci, "Gaussian ring basis functions for the analysis of modulated metasurface antennas," IEEE Trans. Antennas Propag., vol. 63, no. 9, pp. 3982-3993, Sep. 2015. 